PROCEEDINGS OF THE

AMERICAN MATHEMATICAL SOCIETY

Volume 135, Number 8, August 2007, Pages 2607-2617

S 0002-9939(07)08901-0

Article electronically published on March 30, 2007

\title{
HOMOLOGY VANISHING THEOREMS FOR SUBMANIFOLDS
}

\author{
THEODOROS VLACHOS
}

(Communicated by Jon G. Wolfson)

\begin{abstract}
We relate intrinsic and extrinsic curvature invariants to the homology groups of submanifolds in space forms of nonnegative curvature. More precisely, we provide bounds for the squared length of the second fundamental form, or the Ricci curvature in terms of the mean curvature, which force homology to vanish in a range of intermediate dimensions. Moreover, we give examples which show that these conditions are sharp.
\end{abstract}

\section{INTRODUCTION}

It has been a fascinating problem to find out to what extent several restrictions on curvatures of a Riemannian manifold $M$ yield information on the topology of $M$. The same question can be raised from the point of view of submanifold geometry. Namely, it should be interesting to know how the topology of submanifolds of space forms is affected by conditions on intrinsic and extrinsic curvature invariants.

The aim of this paper is to study this question for submanifolds in space forms of nonnegative curvature. Lawson and Simons [4] showed that bounds on the second fundamental form for submanifolds of spheres imply vanishing of homology groups. We recall that Lawson and Simons did this by using the second variation of area to rule out stable minimal currents in certain dimensions. Since one can minimize area in a homology class, this trivializes integral homology. However, the precise bounds in [4, Theorem 4] are technical and hard to understand geometrically. Realizing this, those authors proved that their bounds follow from a simpler absolute bound on the length of the second fundamental form.

In the present paper we provide bounds for the length of the second fundamental form or the Ricci curvature in terms of the mean curvature, for submanifolds in space forms of nonnegative curvature, which force homology to vanish in a range of intermediate dimensions. The choice of these bounds is inspired by the standard immersion of the torus $\mathbb{S}^{k}(r) \times \mathbb{S}^{n-k}(s)$ into the unit sphere $\mathbb{S}^{n+1}$, where $\mathbb{S}^{k}(r)$ denotes the $k$-dimensional sphere of radius $r<1$ and $r^{2}+s^{2}=1$. The shape operator $A$ of $\mathbb{S}^{k}(r) \times \mathbb{S}^{n-k}(s)$ in $\mathbb{S}^{n+1}$, with respect to some unit normal vector field, is given by $A=\frac{s}{r} I_{k} \oplus\left(-\frac{r}{s}\right) I_{n-k}$, where $I_{k}$ and $I_{n-k}$ denote the identity transformations on the tangent bundles of $\mathbb{S}^{k}(r)$ and $\mathbb{S}^{n-k}(s)$, respectively. The principal curvatures are $s / r$ of multiplicity $k$ and $-r / s$ of multiplicity $n-k$,

Received by the editors May 9, 2006.

2000 Mathematics Subject Classification. Primary 53C40; Secondary 53C20.

Key words and phrases. Ricci curvature, length of the second fundamental form, mean curvature, homology groups.

(C)2007 American Mathematical Society Reverts to public domain 28 years from publication 
and consequently the mean curvature $H$ and the squared length $S$ of the second fundamental form are given, respectively, by

$$
H=\frac{k-n r^{2}}{n r s} \text { and } S=\frac{k s^{4}+(n-k) r^{4}}{r^{2} s^{2}} .
$$

By a straightforward computation, we find that

$$
r^{2}=\frac{2 k+n H^{2} \pm|H| \sqrt{n^{2} H^{2}+4 k(n-k)}}{2 n\left(1+H^{2}\right)} .
$$

Substituting this into the above formula for $S$, and carrying out a tedious computation, we see that the squared length of the second fundamental form of $\mathbb{S}^{k}(r) \times$ $\mathbb{S}^{n-k}(s)$ satisfies

$$
S=n+\frac{n^{3} H^{2}}{2 k(n-k)} \pm \frac{n(n-2 k)}{2 k(n-k)}|H| \sqrt{n^{2} H^{2}+4 k(n-k)},
$$

where the sign + or - is chosen according to $r^{2} \leq \frac{k}{n}$, or $r^{2} \geq \frac{k}{n}$, respectively. Moreover, the Ricci curvature of $\mathbb{S}^{k}(r) \times \mathbb{S}^{n-k}(s)$ in the principal directions attains the values $b_{+}(n, k,|H|), b_{-}(n, n-k,|H|)$ if $r^{2} \leq \frac{k}{n}$, or $b_{+}(n, n-k,|H|), b_{-}(n, k,|H|)$ if $r^{2} \geq \frac{k}{n}$, where $b_{ \pm}$is defined by

$$
b_{ \pm}(n, k, t):=\frac{n(k-1)}{k}+\frac{n^{2}(k-1)}{2 k^{2}} t^{2} \pm \frac{n(k-1)}{2 k^{2}} t \sqrt{n^{2} t^{2}+4 k(n-k)}, t \in \mathbb{R} .
$$

We also set

$$
a(n, k, t, c):=n c+\frac{n^{3} t^{2}}{2 k(n-k)}-\frac{n|n-2 k|}{2 k(n-k)} t \sqrt{n^{2} t^{2}+4 k(n-k) c}, t \in \mathbb{R}, c \geq 0 .
$$

This example served as the motivation for our homology vanishing results. The main idea is to show that for submanifolds in space forms of nonnegative curvature $c \geq 0$ one can get the bounds in 4 from bounds on the squared length $S$ of the second fundamental form of the form $S<a(n, k,|\vec{H}|, c)$, or from bounds on the Ricci curvature of the form Ric $>b_{+}(n, k,|\vec{H}|)$, where $|\vec{H}|$ is the length of the mean curvature vector field $\vec{H}$. The key point is that $a(n, k,|\vec{H}|, c)$ and $b_{+}(n, k,|\vec{H}|)$ grow roughly quadratically in $|\vec{H}|$. This can force homology to vanish even in the presence of arbitrary large length of the second fundamental form. Obviously, the absolute bound for the length of the second fundamental form in [4 cannot do that. Specifically, we prove the following results.

Theorem 1. Let $M^{n}, n \geq 4$, be a compact, oriented $n$-dimensional submanifold of the $(n+m)$-dimensional space form $\mathbb{Q}_{c}^{n+m}$ of curvature $c \geq 0$ with mean curvature vector field $\vec{H}$. If the squared length $S$ of the second fundamental form satisfies

$$
S<a(n, k,|\vec{H}|, c)
$$

for an integer $k$ such that $0<k<n$, then $H_{p}\left(M^{n} ; \mathbb{Z}\right)=0$ for all $p \in\{k, \ldots, n-k\} \backslash$ $\{1, n-1\}$, where $H_{p}\left(M^{n} ; \mathbb{Z}\right)$ denotes the $p$-th homology group of $M^{n}$ with integer coefficients.

Corollary 1. Let $M^{n}, n \geq 4$, be a compact, oriented $n$-dimensional submanifold of the space form $\mathbb{Q}_{c}^{n+m}$ of curvature $c \geq 0$ with mean curvature vector field $\vec{H}$. Assume that the squared length $S$ of the second fundamental form satisfies the condition

$$
S<a(n, 2,|\vec{H}|, c)
$$


If the fundamental group $\pi_{1}\left(M^{n}\right)$ is finite, then $M^{n}$ is homeomorphic to $\mathbb{S}^{n}$. In particular, if $M^{n}$ is simply connected, then $M^{n}$ is homeomorphic to $\mathbb{S}^{n}$.

Shiohama and $\mathrm{Xu}$ [5] proved a topological sphere theorem under the condition $S<a(n, 1,|\vec{H}|, c)$. Corollary 1 sharpens their result since it can be easily checked that $a(n, 1,|\vec{H}|, c) \leq a(n, 2,|\vec{H}|, c)$, and equality holds if and only if $n=3$ or $\vec{H}=0$.

It is worth noticing that all hyperspheres and all $H(r)$-tori $\mathbb{S}^{1}(r) \times \mathbb{S}^{n-1}\left(\sqrt{1-r^{2}}\right)$ in $\mathbb{S}^{n+1}$ with $r^{2}>\frac{1}{n}$ do satisfy (1.2). In addition, the torus $\mathbb{S}^{2}(r) \times \mathbb{S}^{n-2}\left(\sqrt{1-r^{2}}\right)$ satisfies $S=a(n, 2,|\vec{H}|, 1)$ if $r^{2} \geq \frac{2}{n}$. Furthermore, Wallach [8] constructed a minimal immersion of an $n$-dimensional complex projective space $\mathbb{C P}^{n}$ of constant holomorphic curvature $2 n /(n+1)$ into the sphere $\mathbb{S}^{n(n+2)-1}$ which satisfies $S=a(2 n, 2,|\vec{H}|, 1)$ if $n=2$. These examples show that the above results are optimal.

Theorem 2. Let $M^{n}, n \geq 4$, be a compact, $n$-dimensional submanifold of the unit sphere $\mathbb{S}^{n+m}$ with mean curvature vector field $\vec{H}$. Assume that the Ricci curvature satisfies

$$
\text { Ric }>b_{+}(n, k,|\vec{H}|),
$$

where $k$ is an integer such that $2 \leq k \leq n / 2$. Then $H_{p}\left(M^{n} ; \mathbb{Z}\right)=H_{n-p}\left(M^{n} ; \mathbb{Z}\right)=0$ for all $1 \leq p \leq k$. Moreover, $M^{n}$ is simply connected.

Corollary 2. Let $M^{n}, n \geq 6$, be a compact, $n$-dimensional submanifold of $\mathbb{S}^{n+m}$ with mean curvature vector field $\vec{H}$. Assume that the Ricci curvature satisfies

$$
\text { Ric }>b_{+}(n,(n-2) / 2,|\vec{H}|)
$$

and $n$ is even. Then either $M^{n}$ is homeomorphic to $\mathbb{S}^{n}$ or $M^{n}$ is simply connected, $H_{p}\left(M^{n} ; \mathbb{Z}\right)=H_{n-p}\left(M^{n} ; \mathbb{Z}\right)=0$ for all $1 \leq p \leq(n-2) / 2$, and $H_{n / 2}\left(M^{n} ; \mathbb{Z}\right)=$ $\mathbb{Z} \oplus \ldots \oplus \mathbb{Z}(r \geq 1$ summands).

Let $M_{m, n-m}=\mathbb{S}^{m}(\sqrt{m / n}) \times \mathbb{S}^{n-m}(\sqrt{(n-m) / n})$ be the Clifford torus in $\mathbb{S}^{n+1}$. It is easy to see that $M_{k, k}$ fulfils (1.4) and $H_{k}\left(M_{k, k} ; \mathbb{Z}\right)=\mathbb{Z} \oplus \mathbb{Z}$. Moreover, $M_{k-1, k+1}$ satisfies Ric $\geq b_{+}(2 k, k-1,|\vec{H}|)$, while $H_{k-1}\left(M_{k-1, k+1} ; \mathbb{Z}\right)=\mathbb{Z}$, and $H_{k}\left(M_{k-1, k+1} ; \mathbb{Z}\right)=0$. Thus Theorem 2 and Corollary 2 are sharp and extend previous results in [1, 3, 7].

\section{Preliminaries}

Let $M^{n}$ be an $n$-dimensional submanifold of codimension $m$ of the $(n+m)$ dimensional space form $\mathbb{Q}_{c}^{n+m}$ of curvature $c$, equipped with the induced Riemannian metric $\langle$,$\rangle . Denote the standard connection of \mathbb{Q}_{c}^{n+m}$ by $\bar{\nabla}$, the Riemannian connection of $M^{n}$ by $\nabla$, and the second fundamental form by $B$. For tangent vectors fields $X$ and $Y$ of $M^{n}$, we have the Gauss formula

$$
\bar{\nabla}_{X} Y=\nabla_{X} Y+B(X, Y)
$$

and the Weingarten formula

$$
\bar{\nabla}_{X} \xi=-A_{\xi} X+D_{X} \xi,
$$

where the $(1,1)$ tensor field $A_{\xi}$ is the shape operator associated with a normal vector field $\xi$, and $D$ is the connection in the normal bundle of $M^{n}$. It is well known that 
$\left\langle A_{\xi} X, Y\right\rangle=\langle B(X, Y), \xi\rangle$. The mean curvature vector field $\vec{H}$ is given by

$$
\vec{H}=\frac{1}{n} \sum_{i=1}^{n} B\left(e_{i}, e_{i}\right)
$$

where $\left\{e_{1}, \ldots, e_{n}\right\}$ is a local orthonormal frame field in the tangent bundle of $M^{n}$, or equivalently

$$
\vec{H}=\frac{1}{n} \sum_{\alpha=n+1}^{n+m}\left(\operatorname{trace} A_{\alpha}\right) e_{\alpha},
$$

where $\left\{e_{n+1}, \ldots, e_{n+m}\right\}$ is a local orthonormal frame field in the normal bundle of $M^{n}$ and $A_{\alpha}$ denotes the shape operator associated with $e_{\alpha}$. The squared length $S$ of the second fundamental form is defined by

$$
S=\sum_{\alpha=n+1}^{n+m} \operatorname{trace}\left(A_{\alpha}^{2}\right)
$$

Moreover, the Ricci curvature in the direction of a unit tangent vector $X$ of $M^{n}$ is given by

$$
\operatorname{Ric}(X)=(n-1) c+\sum_{\alpha=n+1}^{n+m}\left(\operatorname{trace} A_{\alpha}\right)\left\langle A_{\alpha} X, X\right\rangle-\sum_{\alpha=n+1}^{n+m}\left|A_{\alpha} X\right|^{2} .
$$

The main tool for the proofs is the following result due to Lawson and Simons for the nonexistence of stable currents. In fact, the following theorem was proved in 4 for $c>0$. The proof for $c=0$ is similar and was given by Xin 9]. The vanishing of the homology groups below follows from the existence theorems of Federer and Fleming [2] which assert that for any compact Riemannian manifold $M^{n}$, any nontrivial integral homology class in $H_{i}\left(M^{n} ; \mathbb{Z}\right)$ corresponds to a stable integral current.

Theorem 3. Let $M^{n}$ be a compact, n-dimensional submanifold of the space form $\mathbb{Q}_{c}^{n+m}$ of curvature $c \geq 0$ with second fundamental form $B$, and let $p, q$ be positive integers such that $p+q=n$. If for any point $x \in M^{n}$ and any orthonormal basis $\left\{e_{1}, \ldots, e_{p}, \ldots, e_{n}\right\}$ of the tangent space $T_{x} M^{n}$, the inequality

$$
\sum_{i=1}^{p} \sum_{j=p+1}^{n}\left(2\left|B\left(e_{i}, e_{j}\right)\right|^{2}-\left\langle B\left(e_{i}, e_{i}\right), B\left(e_{j}, e_{j}\right)\right\rangle\right)<p q c
$$

is satisfied, then there are no stable p-currents in $M^{n}$. Moreover, $H_{p}\left(M^{n} ; \mathbb{Z}\right)=$ $H_{q}\left(M^{n} ; \mathbb{Z}\right)=0$.

\section{Proofs}

We need the following algebraic lemma for the proof of Theorem 1.

Lemma 1. Let $x_{1}, \ldots, x_{p}, \ldots, x_{n}$ be real numbers and $p, q$ be positive integers such that $p+q=n$. We set $\sigma_{1}=\sum_{r=1}^{n} x_{r}$ and $\sigma_{2}=\sum_{r=1}^{n} x_{r}^{2}$. Then we have

$$
-\sum_{i=1}^{p} x_{i} \sum_{j=p+1}^{n} x_{j} \leq \frac{p q}{n}\left(\sigma_{2}-\frac{2}{n} \sigma_{1}^{2}+\frac{|p-q|}{n \sqrt{p q}}\left|\sigma_{1}\right| \sqrt{n \sigma_{2}-\sigma_{1}^{2}}\right) .
$$


Proof. We apply the Lagrange multiplier method to find the maximum of the function

$$
f\left(x_{1}, \ldots, x_{p}, \ldots, x_{n}\right):=-\sum_{i=1}^{p} x_{i} \sum_{j=p+1}^{n} x_{j}
$$

subject to the constraints

$$
\begin{aligned}
& \sum_{i=1}^{p} x_{i}+\sum_{j=p+1}^{n} x_{j}=\sigma_{1}, \\
& \sum_{i=1}^{p} x_{i}^{2}+\sum_{j=p+1}^{n} x_{j}^{2}=\sigma_{2} .
\end{aligned}
$$

We consider the function

$$
g=f+\lambda\left(\sum_{i=1}^{p} x_{r}+\sum_{j=p+1}^{n} x_{j}-\sigma_{1}\right)+\mu\left(\sum_{i=1}^{p} x_{i}^{2}+\sum_{j=p+1}^{n} x_{j}^{2}-\sigma_{2}\right),
$$

where $\lambda$ and $\mu$ are the Lagrange multipliers. Differentiating with respect to the variables $x_{i}, 1 \leq i \leq p$, and $x_{j}, p+1 \leq j \leq n$, we obtain the following system for the critical points:

$$
\begin{aligned}
& 2 \mu x_{i}+\lambda-\sum_{j=p+1}^{n} x_{j}=0 \text { for } 1 \leq i \leq p, \\
& 2 \mu x_{j}+\lambda-\sum_{i=1}^{p} x_{i}=0 \text { for } p+1 \leq j \leq n .
\end{aligned}
$$

In the case where $\mu=0$, from (3.1), (3.3) and (3.4) we get the critical value $f=-\sigma_{1}^{2} / 4$.

Now assume that $\mu \neq 0$. Then (3.3) and (3.4) imply $x_{i}=u$ for $1 \leq i \leq p$, and $x_{j}=v$ for $p+1 \leq j \leq n$. Furthermore, (3.1) and (3.2) yield

$$
p u+q v=\sigma_{1}, p u^{2}+q v^{2}=\sigma_{2},
$$

from which we find

$$
u=\frac{\sigma_{1}}{n} \pm \frac{\sqrt{p q}}{p n} \sqrt{n \sigma_{2}-\sigma_{1}^{2}}, v=\frac{\sigma_{1}}{n} \mp \frac{\sqrt{p q}}{q n} \sqrt{n \sigma_{2}-\sigma_{1}^{2}},
$$

and consequently we obtain the two critical values

$$
\frac{p q}{n}\left(\sigma_{2}-\frac{2}{n} \sigma_{1}^{2} \pm \frac{|p-q|}{n \sqrt{p q}}\left|\sigma_{1}\right| \sqrt{n \sigma_{2}-\sigma_{1}^{2}}\right)
$$

for $f$. It is easy to verify that the bigger of all critical values is

$$
f=\frac{p q}{n}\left(\sigma_{2}-\frac{2}{n} \sigma_{1}^{2}+\frac{|p-q|}{n \sqrt{p q}}\left|\sigma_{1}\right| \sqrt{n \sigma_{2}-\sigma_{1}^{2}}\right) .
$$

This completes the proof.

Proof of Theorem 1. Take any positive integers $p, q$ such that $p+q=n$. Let $x \in M^{n}$ and $\left\{e_{1}, \ldots, e_{p}, \ldots, e_{n}\right\}$ be an arbitrary orthonormal basis of the tangent space $T_{x} M^{n}$. We choose an orthonormal basis $\left\{e_{n+1}, \ldots, e_{n+m}\right\}$ of the normal space such that 
$\vec{H}=|\vec{H}| e_{n+1}$ at $x$. Denote by $\left(h_{i j}^{\alpha}\right)$ the matrix of the corresponding shape operator $A_{\alpha}, \alpha=n+1, \ldots, n+m$, with respect to the basis $\left\{e_{1}, \ldots, e_{n}\right\}$. Then

$$
\sum_{l=1}^{n} h_{l l}^{\alpha}=0 \text { for } \alpha=n+2, \ldots, n+m
$$

and

$$
\sum_{l=1}^{n} h_{l l}^{n+1}=n|\vec{H}|
$$

Appealing to Lemma 1, we obtain

$$
-\sum_{i=1}^{p} h_{i i}^{\alpha} \sum_{j=p+1}^{n} h_{j j}^{\alpha} \leq \frac{p q}{n} \sum_{l=1}^{n}\left(h_{l l}^{\alpha}\right)^{2} \text { for } \alpha=n+2, \ldots, n+m,
$$

and

$$
\begin{aligned}
& -\sum_{i=1}^{p} h_{i i}^{n+1} \sum_{j=p+1}^{n} h_{j j}^{n+1} \\
\leq & \frac{p q}{n}\left(\sum_{l=1}^{n}\left(h_{l l}^{n+1}\right)^{2}-2 n|\vec{H}|^{2}+\frac{n|p-q|}{\sqrt{n p q}}|\vec{H}| \sqrt{\sum_{l=1}^{n}\left(h_{l l}^{n+1}\right)^{2}-n|\vec{H}|^{2}}\right) .
\end{aligned}
$$

Using these inequalities and bearing in mind that

$$
\begin{aligned}
& \sum_{i=1}^{p} \sum_{j=p+1}^{n}\left(2\left|B\left(e_{i}, e_{j}\right)\right|^{2}-\left\langle B\left(e_{i}, e_{i}\right), B\left(e_{j}, e_{j}\right)\right\rangle\right) \\
= & \sum_{\alpha=n+1}^{n+m}\left(2 \sum_{i=1}^{p} \sum_{j=p+1}^{n}\left(h_{i j}^{\alpha}\right)^{2}-\sum_{i=1}^{p} h_{i i}^{\alpha} \sum_{j=p+1}^{n} h_{j j}^{\alpha}\right),
\end{aligned}
$$

we get

$$
\begin{aligned}
\sum_{i=1}^{p} & \sum_{j=p+1}^{n}\left(2\left|B\left(e_{i}, e_{j}\right)\right|^{2}-\left\langle B\left(e_{i}, e_{i}\right), B\left(e_{j}, e_{j}\right)\right\rangle\right) \\
\leq & 2 \sum_{i=1}^{p} \sum_{j=p+1}^{n} \sum_{\alpha=n+1}^{n+m}\left(h_{i j}^{\alpha}\right)^{2}+\frac{p q}{n} \sum_{\alpha=n+2}^{n+m} \sum_{l=1}^{n}\left(h_{l l}^{\alpha}\right)^{2} \\
& +\frac{p q}{n}\left(\sum_{l=1}^{n}\left(h_{l l}^{n+1}\right)^{2}-2 n|\vec{H}|^{2}+\frac{n|p-q|}{\sqrt{n p q}}|\vec{H}| \sqrt{\sum_{l=1}^{n}\left(h_{l l}^{n+1}\right)^{2}-n|\vec{H}|^{2}}\right) .
\end{aligned}
$$

Now we suppose that $1<p<n-1$. We claim that $p q \geq n$. In fact, this is trivial for $n=4$, while for $n>4$ it is enough to see that $t(n-t) \geq n$ for all $2 \leq t \leq n-2$. Then on account of $p q \geq n$, the above inequality yields

$$
\begin{aligned}
& \sum_{i=1}^{p} \sum_{j=p+1}^{n}\left(2\left|B\left(e_{i}, e_{j}\right)\right|^{2}-\left\langle B\left(e_{i}, e_{i}\right), B\left(e_{j}, e_{j}\right)\right\rangle\right) \\
& \quad \leq \frac{p q}{n}\left(S-2 n|\vec{H}|^{2}+\frac{n|p-q|}{\sqrt{n p q}}|\vec{H}| \sqrt{S-n|\vec{H}|^{2}}\right)
\end{aligned}
$$


A direct computation shows that condition (1.1) is equivalent to

$$
\sqrt{S-n|\vec{H}|^{2}}<\sqrt{C(n, k,|\vec{H}|, c)}
$$

where $C(n, k,|\vec{H}|, c)$ is given by

$$
C(n, k,|\vec{H}|, c)=\frac{n}{4 k(n-k)}\left(\sqrt{n^{2}|\vec{H}|^{2}+4 k(n-k) c}-|n-2 k||\vec{H}|\right)^{2} .
$$

We now consider the polynomial

$$
P(t)=t^{2}+\frac{n|n-2 k|}{\sqrt{n k(n-k)}}|\vec{H}| t-n\left(c+|\vec{H}|^{2}\right), t \in \mathbb{R} .
$$

Since $\sqrt{C(n, k,|\vec{H}|, c)}$ is the positive root of $P(t)=0$, inequality $\sqrt{S-n|\vec{H}|^{2}}<$ $\sqrt{C(n, k,|\vec{H}|, c)}$ is equivalent to $P\left(\sqrt{S-n|\vec{H}|^{2}}\right)<0$. Thus condition (1.1) is equivalent to

$$
S<n c+2 n|\vec{H}|^{2}-\frac{n|n-2 k|}{\sqrt{n k(n-k)}}|\vec{H}| \sqrt{S-n|\vec{H}|^{2}} .
$$

Hence we obtain

$$
\begin{aligned}
\sum_{i=1}^{p} \sum_{j=p+1}^{n}\left(2\left|B\left(e_{i}, e_{j}\right)\right|^{2}-\left\langle B\left(e_{i}, e_{i}\right), B\left(e_{j}, e_{j}\right)\right\rangle\right)<p q c \\
+\frac{p q}{\sqrt{n}}|\vec{H}| \sqrt{S-n|\vec{H}|^{2}}\left(\frac{|n-2 p|}{\sqrt{p(n-p)}}-\frac{|n-2 k|}{\sqrt{k(n-k)}}\right) .
\end{aligned}
$$

We claim that inequality (2.2) is satisfied. If $n=2 p=2 k$, then (3.5) immediately implies (2.2). Assume now that either $n \neq 2 p$ or $n \neq 2 k$. Then (3.5) can be written as

$$
\begin{aligned}
& \sum_{i=1}^{p} \sum_{j=p+1}^{n}\left(2\left|B\left(e_{i}, e_{j}\right)\right|^{2}-\left\langle B\left(e_{i}, e_{i}\right), B\left(e_{j}, e_{j}\right)\right\rangle\right)<p q c \\
& \quad+\frac{p q n^{2}(k-p)(n-k-p)|\vec{H}| \sqrt{S-n|\vec{H}|^{2}}}{\sqrt{n p q k(n-k)}(|n-2 p| \sqrt{k(n-k)}+|n-2 k| \sqrt{p(n-p)})} .
\end{aligned}
$$

Therefore (2.2) is fulfilled for all $p=k, \ldots, n-k$, and according to Theorem 3 , we obtain $H_{p}\left(M^{n} ; \mathbb{Z}\right)=0$ for all $p \in\{k, \ldots, n-k\} \backslash\{1, n-1\}$, which completes the proof of the theorem.

Proof of Corollary 1. By virtue of Theorem 1 , we deduce that $H_{p}\left(M^{n} ; \mathbb{Z}\right)=0$ for all $2 \leq p \leq n-2$. Suppose that the fundamental group $\pi_{1}\left(M^{n}\right)$ is finite. From $H_{n-2}\left(M^{n} ; \mathbb{Z}\right)=0$ and the universal coefficient theorem, we infer that $H^{n-1}\left(M^{n} ; \mathbb{Z}\right)$ has no torsion and neither does $H_{1}\left(M^{n} ; \mathbb{Z}\right)$ by Poincaré duality. Since $\pi_{1}\left(M^{n}\right)$ is finite, we have $H_{1}\left(M^{n} ; \mathbb{Z}\right)=0$ and $H_{n-1}\left(M^{n} ; \mathbb{Z}\right)=0$. So $M^{n}$ is a homology sphere. We consider the universal Riemannian covering $\widetilde{M}^{n}$ of $M^{n}$. Since $\pi_{1}\left(M^{n}\right)$ is finite, $\widetilde{M}^{n}$ is compact. Obviously $\widetilde{M}^{n}$ satisfies condition (1.2) and by virtue of Theorem 1 , we get $H_{p}\left(\widetilde{M^{n}} ; \mathbb{Z}\right)=0$ for all $2 \leq p \leq n-2$. Moreover, $\widetilde{M}^{n}$ is a homology sphere with fundamental group $\pi_{1}\left(\widetilde{M}^{n}\right)=0$. Therefore $\widetilde{M}^{n}$ is a homotopy sphere. By the generalized Poincaré conjecture (Smale $n \geq 5$, Freedman $n=4$ ) we deduce that $\widetilde{M}^{n}$ 
is homeomorphic to $\mathbb{S}^{n}$. Since the homology sphere $M^{n}$ is covered by $\mathbb{S}^{n}$, a result due to Sjerve [6] implies that $\pi_{1}\left(M^{n}\right)=0$ and consequently $M^{n}$ is homeomorphic to $\mathbb{S}^{n}$. This completes the proof.

The following is needed for the proof of Theorem 2 .

Lemma 2. Let $M^{n}, n \geq 4$, be an $n$-dimensional submanifold of $\mathbb{S}^{n+m}$ with mean curvature vector field $\vec{H}$. Assume that $M^{n}$ satisfies condition (1.3) for an integer $k$ such that $0<k \leq n / 2$. Then $n^{2}(k-2)|\vec{H}|^{2}<4(n-k)$.

Proof. Since the desired inequality is obvious for $n=4$, we may assume that $n>4$ and $k>2$. In view of (1.3), the scalar curvature $\tau$ of $M^{n}$ satisfies $\tau>n b_{+}(n, k,|\vec{H}|)$. Moreover, from the Gauss equation $\tau=n^{2}|\vec{H}|^{2}-S+n(n-1)$, we clearly have $\tau \leq n^{2}|\vec{H}|^{2}+n(n-1)$. Thus we obtain $n|\vec{H}|^{2}+n-1>b_{+}(n, k,|\vec{H}|)$, or equivalently

$$
2 k(n-k)+n|\vec{H}|^{2}\left(2 k^{2}-n k+n\right)>n(k-1)|\vec{H}| \sqrt{n^{2}|\vec{H}|^{2}+4 k(n-k)} .
$$

After squaring, we get

$$
n^{2}\left(k^{2}-n k+n\right)|\vec{H}|^{4}+n(n-k)(2 k-n k+n)|\vec{H}|^{2}+(n-k)^{2}>0 .
$$

We note that $t^{2}-n t+n<0$ for all $2 \leq t \leq n-2$. Then $k^{2}-n k+n<0$ since $2<k<n-2$, and the above inequality yields

$$
|\vec{H}|^{2}<\frac{(n-k)(2 k-n(k-1)+(k-1) \sqrt{n(n-4)})}{2 n\left(n k-k^{2}-n\right)} .
$$

Therefore in order to prove the desired inequality, it is enough to show that

$$
\frac{2 k-n(k-1)+(k-1) \sqrt{n(n-4)}}{2\left(n k-k^{2}-n\right)}<\frac{4}{n(k-2)},
$$

or equivalently $g(k)<0$, where $g$ is the polynomial given by

$g(t):=(n \sqrt{n}-(n+2) \sqrt{n-4}) t^{2}-3 n(\sqrt{n}-\sqrt{n-4}) t+2 n(\sqrt{n}-\sqrt{n-4}), t \in \mathbb{R}$.

We note that $g(1)<0$. In addition, it follows easily that

$$
g\left(\frac{n}{2}\right)=\frac{n}{4}\left(\left(n^{2}-6 n+8\right) \sqrt{n}-\left(n^{2}-4 n+8\right) \sqrt{n-4}\right)<0 .
$$

Hence $g(k)<0$ for $1 \leq k \leq n / 2$, which completes the proof of the lemma.

Proof of Theorem 2. Let $p, q$ be positive integers such that $p+q=n, x$ is an arbitrary point of $M^{n}$ and $\left\{e_{1}, \ldots, e_{p}, \ldots, e_{n}\right\}$ is an arbitrary orthonormal basis of the tangent space $T_{x} M^{n}$. We choose an orthonormal basis $\left\{e_{n+1}, \ldots, e_{n+m}\right\}$ of the normal space such that $\vec{H}=|\vec{H}| e_{n+1}$ at $x$. Denote by $A_{\alpha}, \alpha=n+1, \ldots, n+m$, the corresponding shape operators. Then we have

$$
\begin{aligned}
& \sum_{i=1}^{p} \sum_{j=p+1}^{n}\left(2\left|B\left(e_{i}, e_{j}\right)\right|^{2}-\left\langle B\left(e_{i}, e_{i}\right), B\left(e_{j}, e_{j}\right)\right\rangle\right) \\
= & 2 \sum_{i=1}^{p} \sum_{j=p+1}^{n}\left|B\left(e_{i}, e_{j}\right)\right|^{2}-n \sum_{i=1}^{p}\left\langle B\left(e_{i}, e_{i}\right), \vec{H}\right\rangle+\sum_{i, j=1}^{p}\left\langle B\left(e_{i}, e_{i}\right), B\left(e_{j}, e_{j}\right)\right\rangle,
\end{aligned}
$$


or equivalently

$$
\begin{aligned}
& \sum_{i=1}^{p} \sum_{j=p+1}^{n}\left(2\left|B\left(e_{i}, e_{j}\right)\right|^{2}-\left\langle B\left(e_{i}, e_{i}\right), B\left(e_{j}, e_{j}\right)\right\rangle\right) \\
& \quad=2 \sum_{i=1}^{p} \sum_{j=p+1}^{n} \sum_{\alpha=n+1}^{n+m}\left\langle A_{\alpha} e_{i}, e_{j}\right\rangle^{2}-n|\vec{H}| \sum_{i=1}^{p}\left\langle A_{n+1} e_{i}, e_{i}\right\rangle+\sum_{\alpha=n+1}^{n+m}\left(\sum_{i=1}^{p}\left\langle A_{\alpha} e_{i}, e_{i}\right\rangle\right)^{2} .
\end{aligned}
$$

By Cauchy-Schwarz inequality we obtain

$$
\begin{aligned}
& \text { (3.6) } \sum_{i=1}^{p} \sum_{j=p+1}^{n}\left(2\left|B\left(e_{i}, e_{j}\right)\right|^{2}-\left\langle B\left(e_{i}, e_{i}\right), B\left(e_{j}, e_{j}\right)\right\rangle\right) \\
& \leq 2 \sum_{i=1}^{p} \sum_{j=p+1}^{n} \sum_{\alpha=n+1}^{n+m}\left\langle A_{\alpha} e_{i}, e_{j}\right\rangle^{2}-n|\vec{H}| \sum_{i=1}^{p}\left\langle A_{n+1} e_{i}, e_{i}\right\rangle+p \sum_{\alpha=n+1}^{n+m} \sum_{i=1}^{p}\left\langle A_{\alpha} e_{i}, e_{i}\right\rangle^{2} .
\end{aligned}
$$

Bearing in mind the choice of $e_{n+1}$, our assumption (1.3) and (2.1), we verify that each eigenvalue $\lambda$ of $A_{n+1}$ satisfies $\lambda^{2}-n|\vec{H}| \lambda+b_{+}(n, k,|\vec{H}|)-n+1<0$. Hence we have

$$
\lambda<\frac{1}{2}\left(n|\vec{H}|+\frac{1}{k}|-n(k-1)| \vec{H}\left|+\sqrt{n^{2} \mid \vec{H}}\right|^{2}+4 k(n-k) \mid\right) .
$$

On account of Lemma 2 , we get $-n(k-1)|\vec{H}|+\sqrt{n^{2}|\vec{H}|^{2}+4 k(n-k)}>0$. Then

and therefore

$$
\lambda<\frac{1}{2 k}\left(n|\vec{H}|+\sqrt{n^{2}|\vec{H}|^{2}+4 k(n-k)}\right)
$$

$$
\left\langle A_{n+1} X, X\right\rangle<\frac{1}{2 k}\left(n|\vec{H}|+\sqrt{n^{2}|\vec{H}|^{2}+4 k(n-k)}\right)
$$

for each unit tangent vector $X$.

Assume that $p=1$. Then (3.6) implies

$$
\sum_{j=2}^{n}\left(2\left|B\left(e_{1}, e_{j}\right)\right|^{2}-\left\langle B\left(e_{1}, e_{1}\right), B\left(e_{j}, e_{j}\right)\right\rangle\right) \leq 2 \sum_{\alpha=n+1}^{n+m}\left|A_{\alpha} e_{1}\right|^{2}-n|\vec{H}|\left\langle A_{n+1} e_{1}, e_{1}\right\rangle
$$

and appealing to $(2.1)$ we obtain

$\sum_{j=2}^{n}\left(2\left|B\left(e_{1}, e_{j}\right)\right|^{2}-\left\langle B\left(e_{1}, e_{1}\right), B\left(e_{j}, e_{j}\right)\right\rangle\right) \leq 2\left(n-1-\operatorname{Ric}\left(e_{1}\right)\right)+n|\vec{H}|\left\langle A_{n+1} e_{1}, e_{1}\right\rangle$.

In view of our assumption (1.3) and (3.7), the above inequality yields

$$
\begin{aligned}
& \sum_{j=2}^{n}\left(2\left|B\left(e_{1}, e_{j}\right)\right|^{2}-\left\langle B\left(e_{1}, e_{1}\right), B\left(e_{j}, e_{j}\right)\right\rangle\right) \\
& <\frac{2(n-k)}{k}+\frac{2-k}{2 k^{2}} n^{2}|\vec{H}|^{2}+\frac{2-k}{2 k^{2}} n|\vec{H}| \sqrt{n^{2}|\vec{H}|^{2}+4 k(n-k)} \\
& \leq \frac{2(n-k)}{k} \leq n-1,
\end{aligned}
$$

since $k \geq 2$. According to Theorem $3, H_{1}\left(M^{n} ; \mathbb{Z}\right)=0$ and there are no stable 1-currents. Using the well known fact that in each nontrivial free homotopy class there exists a length minimizing curve, we deduce that $M^{n}$ is simply connected. 
Now assume that $p \geq 2$. Then (3.6) implies

$$
\begin{aligned}
& \sum_{i=1}^{p} \sum_{j=p+1}^{n}\left(2\left|B\left(e_{i}, e_{j}\right)\right|^{2}-\left\langle B\left(e_{i}, e_{i}\right), B\left(e_{j}, e_{j}\right)\right\rangle\right) \\
\leq & p \sum_{i=1}^{p} \sum_{\alpha=n+1}^{n+m}\left|A_{\alpha} e_{i}\right|^{2}-n|\vec{H}| \sum_{i=1}^{p}\left\langle A_{n+1} e_{i}, e_{i}\right\rangle
\end{aligned}
$$

and appealing to (2.1), we obtain

$$
\begin{aligned}
& \sum_{i=1}^{p} \sum_{j=p+1}^{n}\left(2\left|B\left(e_{i}, e_{j}\right)\right|^{2}-\left\langle B\left(e_{i}, e_{i}\right), B\left(e_{j}, e_{j}\right)\right\rangle\right) \\
\leq & p \sum_{i=1}^{p}\left(n-1-\operatorname{Ric}\left(e_{i}\right)\right)+n(p-1)|\vec{H}| \sum_{i=1}^{p}\left\langle A_{n+1} e_{i}, e_{i}\right\rangle .
\end{aligned}
$$

Using our assumption (1.3) and (3.7), the above inequality yields

$$
\begin{aligned}
& \sum_{i=1}^{p} \sum_{j=p+1}^{n}\left(2\left|B\left(e_{i}, e_{j}\right)\right|^{2}-\left\langle B\left(e_{i}, e_{i}\right), B\left(e_{j}, e_{j}\right)\right\rangle\right)<\frac{p^{2}(n-k)}{k} \\
& +\frac{p(p-k)}{2 k^{2}} n^{2}|\vec{H}|^{2}+\frac{p(p-k)}{2 k^{2}} n|\vec{H}|\left(n|\vec{H}|+\sqrt{n^{2}|\vec{H}|^{2}+4 k(n-k)}\right) .
\end{aligned}
$$

Then for $2 \leq p \leq k$, we get

$$
\sum_{i=1}^{p} \sum_{j=p+1}^{n}\left(2\left|B\left(e_{i}, e_{j}\right)\right|^{2}-\left\langle B\left(e_{i}, e_{i}\right), B\left(e_{j}, e_{j}\right)\right\rangle\right)<\frac{p^{2}(n-k)}{k} \leq p q .
$$

Appealing again to Theorem 3 , we obtain $H_{p}\left(M^{n} ; \mathbb{Z}\right)=H_{n-p}\left(M^{n} ; \mathbb{Z}\right)=0$ for all $2 \leq p \leq k$, and the theorem is proved.

Proof of Corollary 2. Theorem 2 and our assumption (1.4) imply that $M^{n}$ is simply connected and $H_{p}\left(M^{n} ; \mathbb{Z}\right)=H_{n-p}\left(M^{n} ; \mathbb{Z}\right)=0$ for all $1 \leq p \leq(n-2) / 2$. From the universal coefficient theorem, we infer that $H^{n / 2}\left(M^{n} ; \mathbb{Z}\right)$ has no torsion and neither does $H_{n / 2}\left(M^{n} ; \mathbb{Z}\right)$ by Poincaré duality. Thus $H_{n / 2}\left(M^{n} ; \mathbb{Z}\right)$ is a free abelian group of rank $r \geq 0$. If $r=0$, then $M^{n}$ is a homology sphere with fundamental group $\pi_{1}\left(M^{n}\right)=0$. Hence $M^{n}$ is a homotopy sphere. By the generalized Poincaré conjecture $M^{n}$ is homeomorphic to $\mathbb{S}^{n}$. In the case where $r>0$, we have $H_{n / 2}\left(M^{n} ; \mathbb{Z}\right)=\mathbb{Z} \oplus \ldots \oplus \mathbb{Z}(r \geq 1$ summands $)$. This completes the proof.

\section{REFERENCES}

[1] K.D. Elworthy and S. Rosenberg, Homotopy and homology vanishing theorems and the stability of stochastic flows, Geom. Funct. Anal. 6 (1996), 51-78. MR.1371231 (97a:58198)

[2] H. Federer and W.H. Fleming, Normal and integral currents, Ann. of Math. (2) 72 (1960), 458-520. MR0123260 (23:A588)

[3] Th. Hasanis and Th. Vlachos, Ricci curvature and minimal submanifolds, Pacific J. Math. 197 (2001), 13-24. MR1810205 (2001j:53077)

[4] H.B. Lawson and J. Simons, On stable currents and their application to global problems in real and complex geometry, Ann. of Math. (2) 98 (1973), 427-450. MR0324529(48:2881)

[5] K. Shiohama and $\mathrm{H}$. Xu, The topological sphere theorem for complete submanifolds, Compositio Math. 107 (1997), 221-232. MR.1458750(98i:53080)

[6] D. Sjerve, Homology spheres which are covered by spheres, J. London Math. Soc. (2) 6 (1973), 333-336. MR0310895 (46:9993) 
[7] Th. Vlachos, A sphere theorem for odd-dimensional submanifolds of spheres, Proc. Amer. Math. Soc. 130 (2002), 167-173. MR1855635 (2003c:53083)

[8] N.R. Wallach, Minimal immersions of symmetric spaces into spheres, "Symmetric spaces", Ed. Boothby and Weiss, Dekker, New York, 1972, pp. 1-40. MR0407774 (53:11545)

[9] Y.L. Xin, An application of integral currents to the vanishing theorems, Sci. Sinica Ser. A 27 (1984), 233-241. MR763966 (86b:49060)

Department of Mathematics, University of Ioannina, 45110 Ioannina, Greece

E-mail address: tvlachos@uoi.gr 